\title{
Compositional patterns of ruderal herbs in Santiago, Chile
}

\section{Patrones composicionales de hierbas ruderales en Santiago, Chile}

\author{
Elsa Gärtner ${ }^{1 *}$, Gloria Rojas ${ }^{1} \&$ Sergio A. Castro ${ }^{2,3}$ \\ ${ }^{1}$ Museo Nacional de Historia Natural, Área Botánica, Interior Parque Quinta Normal S/N, Casilla 787, Santiago Chile. \\ 2Laboratorio de Ecología y Biodiversidad, Departamento de Biología, Universidad de Santiago de Chile. Casilla 40, Correo 33, \\ Santiago. Avenida Libertador Bernardo O’Higgins 3363, Santiago, Chile. \\ ${ }^{3}$ Centro para el Desarrollo de la Nanociencia y la Nanotecnología, CEDENNA, 917-0124. Avenida Libertador Bernardo \\ O'Higgins 3363, Santiago, Chile. \\ *elsaagartner@gmail.com
}

\begin{abstract}
Fragmentation of the natural environment is a consequence of urbanisation. It impacts the biodiversity of native flora that characterises a region. This study focused on characterising the diversity, composition and distribution of native and alien ruderal species present in different suburbs of Santiago de Chile. We found that plant assemblages of ruderal species were characterised by a higher proportion of alien species ( 69 taxa), whose original distribution corresponds to the Mediterranean Basin (46 taxa), and a low representation of native species (14 taxa). The results show that the spatial distribution of weeds within Santiago was not random, because two clusters were found based on patterns of compositional similarity. Further research should be undertaken to determine the cause of this phenomenon that probably obeys historical and ecological factors such as the past use of soils or urban landscape ornamentation programs.
\end{abstract}

KEYWORDS: Alien plants, native plants, plant assemblages, weeds.

\begin{abstract}
RESUMEN
La expansión de los centros urbanos tiene como consecuencia la fragmentación de los ambientes naturales y el consecuente impacto en la biodiversidad de la flora nativa que caracteriza a una región. El presente estudio se enfocó en la caracterización de la diversidad, composición y distribución de especies ruderales nativas e introducidas presentes en diferentes comunas de Santiago de Chile. Se encontró que los ensambles de especies ruderales están dominados por la presencia de especies introducidas (69 taxa), cuya distribución original corresponde a la Cuenca Mediterránea (46 taxa), con una baja representación de especies nativas (14 taxa). Los resultados indican además que la distribución espacial de malezas en la ciudad de Santiago no es aleatoria, pues dos conglomerados fueron encontrados en función de los patrones de similitud composicional. Las causas de este fenómeno deben ser investigadas, pero probablemente obedecen a un complejo de factores entre los que se pueden mencionar el modo de uso de suelo, y/o programas de ornamentación del paisaje urbano.
\end{abstract}

Palabras clave: Plantas introducidas, plantas nativas, ensambles, malezas.

\section{INTRODUCTION}

Urbanisation is a common trend and an important part of the transformation of natural landscapes, both at local and global scales (Goddard et al. 2009, Pickett et al. 2001). A consequence of urbanisation is the modification of native flora biodiversity and associated ecological processes both within the cities and their surroundings (Goddard et al. 2009, McDonald et al. 2008, Czech \& Krausman 1997). Recent studies have shown that the distribution of alien species is directly related to the presence of urban centres and street density (Arroyo et al. 2000, Rapoport 1993). Also, the composition of plants within cities is influenced by factors that operate at different scales (Goddard et al. 2009). These factors are directly related to globalisation processes, such as a higher frequency of social, cultural and economical exchanges between continents. These processes contribute to the introduction of alien species, accidental or intentionally, for example the introduction of ornamental plants (Kowarik \& Lippe 2007, McKinney 2006, Mack \& Lonsdale 2001). Alternatively, there are factors stimulating the persistence of native flora, such as native species being 
more adapted to local conditions, or stimulating extinction of native plants competing with more resistant invasive species (McKinney 2006).

Consequently, the floristic composition inside cities is composed of a variety of native and alien species with differences in forms of life, evolution and biogeographic origins (Kowarik 2008). It is also important to highlight that urban flora tend to be more diverse (i.e. number of species) than the rural zones (Kühn et al. 2004). Most of the studies related to urban flora have been carried out on northern hemisphere urban centres, particularly Europe (Albrecht \& Haider 2013, Capotorti et al. 2013, Gregor et al. 2012, Ricotta et al. 2012, Lososová et al. 2011), Asia (Nagendra \& Gopal 2011, Wang et al. 2011) and North America (Knapp et al. 2012). These studies have mostly focused their attention on determining the abundance and distribution of species present within these urban centres.

Urban flora studies in South America (MacGregor-Fors \& Ortega-Alvarez 2013), and particularly in Chile, have been undertaken mostly for land planning and management (Cursach et al. 2012) with some emphasis in creating a floristic catalogue of woody species with ornamental value (Reyes-Paecke \& Meza 2011, Hernández 2007, Escobedo et al. 2006, De la Maza et al. 2002, Hoffmann 1983). For this reason only a few studies on ruderal species are available with most of these species being herbaceous and usually considered weeds (Matthei et al. 1995, De Vallejo 1980). Studies upon diversity of native or alien ruderal species in Chile are scarce within cities. More work is needed to get a better understanding of the ecology of the species that coexist in urban areas.

The current study is an attempt to characterize distributional patterns of ruderal plants in Santiago. It is the first time this approach has been taken and adds important information to global knowledge. This study aims to document the distribution and relative representation of native and alien ruderal species in streets and sidewalks in different suburbs of Santiago, Chile. It also aims to determine if there is a distributional pattern within the city.

\section{MATERIALS AND METHODS}

\section{SAMPLE AREA}

Santiago de Chile $\left(33^{\circ} 26^{\prime} 16^{\prime \prime} \mathrm{S} ; 7^{\circ} 39^{\prime} 01^{\prime \prime} \mathrm{O}\right)$ is located in the middle depression in the central zone of the country, between Cordillera de los Andes and Cordillera de la Costa, at 500 to $600 \mathrm{~m}$ above sea level. The city was founded in 1541 and its population and size has increased exponentially, as consequence, its natural surroundings have deteriorated overtime. The current size of the city covers approximately $600 \mathrm{~km}^{2}$, and has 5.5 million inhabitants (INE, 2002). Santiago's climate is Mediterranean, characterised by rainy winters and dry summers (Luebert \& Pliscoff 2006).
SAMPLING METHODOLOGY

Forty-one sampling sites were randomly located within the city (Fig. 1). Some suburbs not were represented in the sampling. At each site, ruderal herbs were collected along a $100 \mathrm{~m}$ line covering the sidewalk/pavement. Cultivated species were not recorded. All specimens were brought to the Museo Nacional de Historia Natural (Santiago, Chile) for taxonomic determination, and deposited in its herbaria (SGO). Taxonomic identification and biogeographic origin were recorded using De Vallejo (1980), Matthei et al. (1995) and Instituto de Botánica Darwinion (2011). Prevalence was calculated as the percentage of transects occupied for each species.

We analysed the diversity and compositional similarity among suburbs, grouping the transects belonging to the same commune. Then, a matrix was built, indicating presence and absence coded with 0 and 1, respectively. The compositional similarity was then calculated using Jaccard index ( $J$, Jaccard 1908). We built a similarity dendrogram using the Unweighted Pair Group Method with Arithmetic Mean (UPGMA; McGarigal et al. 2000). In order to identify suburb groups with a high compositional similarity, we recalculated 1,000 similarity pseudo-values for the original distribution matrix (Manly, 2006). Using the distribution of frequency of these 1,000 pseudo-values, we identified the critic value for a non-random distribution with $p<0.05$ (Manly 2006). This data was analysed using Poptools 3.2 for Excel.

\section{RESULTS}

The samples included 649 specimens collected from 41 sample sites (Table I).

A total of 95 taxa were found, from which 85 were identified at species or genus level (Table I); the other 10 taxa could not be determined. In total, 24 families were recognized (Table I), the most common being Asteraceae (17 taxa), Poaceae (12 taxa), Fabaceae (seven taxa), Malvaceae and Oxalidaceae (six taxa), and Brassicaceae (five taxa), all of which represented $62.3 \%$ of all species found (see Fig. 2).

Species that were found in more than $50 \%$ of the transects were Erodium moschatum L'Hér. ex Aiton (prevalence $=53.6 \%$ ), Lepidium strictum (S.Watson) Rattan (prevalence $=63.4 \%$ ), Taraxacum officinale F.H. Wigg. (prevalence $=63.4 \%)$, Hordeum murinum L. $($ prevalence $=$ $68.3 \%$ ), Sisymbrium irio L. (prevalence $=70.7 \%$ ), Chamomilla suaveolens (Pursh) Rydb. (prevalence= $75.6 \%$ ), Polygonum aviculare L. (prevalence $=75.61 \%$ ) and Poa annua L. (prevalence $=90.2 \%$ ) (Table I). Another 38 species were found in less than $5 \%$ percent of the transects (Table I). Alien herbs showed higher prevalence than native ones $\left(\chi^{2}=7.4, p<0.05\right)$. 


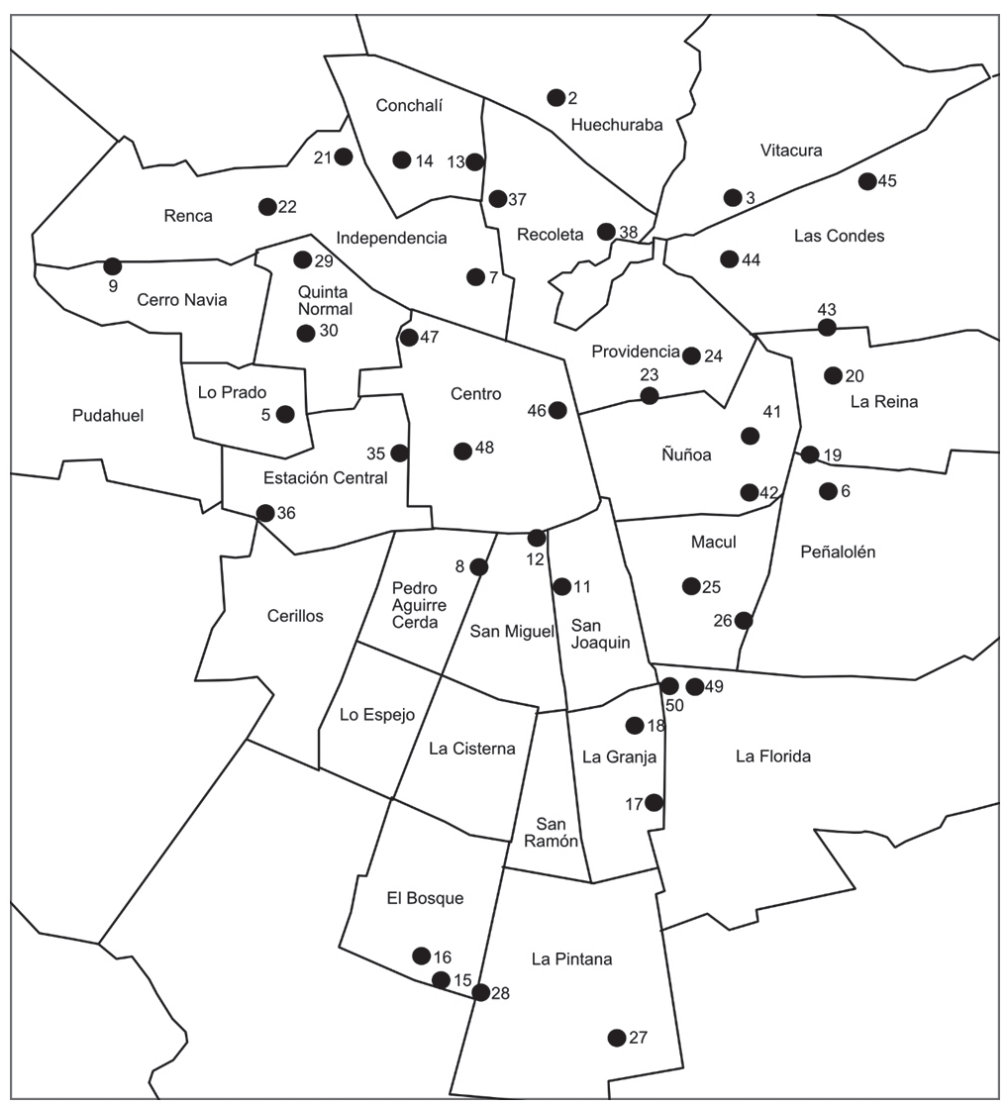

FigURE 1. Location of Santiago city and sampling sites. The sampling sites were arbitrarily numbered.

Figura 1. Ubicación de la ciudad de Santiago y los sitios de muestreo. Los sitios de muestreo fueron arbitrariamente numerados.

TABLE I. Origin and prevalence of collected species $(\times 100)$. Prevalence has been calculated as number of sites where a species has been found divided by the total number of sampling sites (41).

TABLA I. Origen y prevalencia de las especies recolectadas $(\times 100)$. La prevalencia se ha calculado como número de sitios en el cual se ha encontrado la especie divido por el número total de sitios (41).

\begin{tabular}{|c|c|c|c|c|c|}
\hline Species & FAMILY & ORIGIN & ORIGINAL DISTRIBUTIONS & $\begin{array}{c}\text { NuMBER } \\
\text { OF PLANTS } \\
\text { COLLECTED }\end{array}$ & $\begin{array}{l}\text { PreVALENCE } \\
\quad(\times 100)\end{array}$ \\
\hline Amaranthus deflexus $\mathrm{L}$. & Amaranthaceae & Alien & America & 15 & 36.5 \\
\hline Anthriscus caucalis M.Bieb. & Apiaceae & Alien & Mediterranean Basin & 1 & 2.4 \\
\hline Avena barbata Pott ex Link & Poaceae & Alien & Mediterranean Basin & 1 & 2.4 \\
\hline Beta vulgaris $\mathrm{L}$. & Amaranthaceae & Alien & Mediterranean Basin & 1 & 2.4 \\
\hline Brassica nigra $\mathrm{L}$. & Brassicaceae & Alien & Mediterranean Basin & 6 & 14.6 \\
\hline Brassica rapa $\mathrm{L}$. & Brassicaceae & Alien & Eurasian & 1 & 2.4 \\
\hline Bromus catharticus Vahl & Poaceae & Native & & 8 & 19.5 \\
\hline Bromus racemosus L. & Poaceae & Alien & Mediterranean Basin & 4 & 9.7 \\
\hline Carduus pycnocephalus L. & Asteraceae & Alien & Mediterranean Basin & 3 & 7.3 \\
\hline Cerastium glomeratum Thuill. & Caryophyllaceae & Alien & Mediterranean Basin & 2 & 4.8 \\
\hline Cestrum parqui L'Hér. & Solanaceae & Native & & 1 & 2.4 \\
\hline Chamomilla recutita (L.) Rauschert & Asteraceae & Alien & Mediterranean Basin & 4 & 9.7 \\
\hline Chamomilla suaveolens (Pursh) Rydb. & Asteraceae & Alien & North America & 31 & 75.6 \\
\hline
\end{tabular}




\begin{tabular}{|c|c|c|c|c|c|}
\hline SPecies & & ORIGIN & ORIGINAL DISTRIBUTIONS & $\begin{array}{c}\text { NuMBER } \\
\text { OF PLANTS } \\
\text { COLLECTED }\end{array}$ & $\begin{array}{l}\text { Prevalence } \\
\qquad(\times 100)\end{array}$ \\
\hline $\begin{array}{l}\text { Chenopodiastrum murale (L.) S. } \\
\text { Fuentes. Uotila \& Borsch }\end{array}$ & Chenopodiaceae & Alien & Asia minor & 8 & 19.5 \\
\hline Chenopodium album $\mathrm{L}$. & Chenopodiaceae & Alien & Mediterranean Basin & 7 & 17.0 \\
\hline Chenopodium vulvaria L. & Chenopodiaceae & Alien & Eurasian & 1 & 2.4 \\
\hline Convolvulus arvensis $\mathrm{L}$. & Convolvulaceae & Alien & Eurasian & 7 & 17.0 \\
\hline Conyza sp. & Asteraceae & Alien & World temperate zones & 1 & 2.4 \\
\hline Coriandrum sativum L. & Apiaceae & Alien & Mediterranean Basin & 1 & 2.4 \\
\hline Cotula australis Hook.f. & Asteraceae & Alien & Oceania & 16 & 39.0 \\
\hline $\begin{array}{l}\text { Cyclospermum leptophyllum (Pers.) } \\
\text { Sprague }\end{array}$ & Apiaceae & Native & & 2 & 4.8 \\
\hline Cynodon dactylon (L.) Pers. & Poaceae & Alien & $\begin{array}{l}\text { Africa and South- } \\
\text { Europe }\end{array}$ & 19 & 46.3 \\
\hline Dactylis glomerata L. & Poaceae & Alien & Mediterranean Basin & 1 & 2.4 \\
\hline Dichondra sericea Sw. & Convolvulaceae & Native & & 6 & 14.6 \\
\hline $\begin{array}{l}\text { Dysphania ambrosioides (L.) } \\
\text { Mosyakin \& Clemants }\end{array}$ & Chenopodiaceae & Native & & 1 & 2.4 \\
\hline Erodium moschatum L'Hér. ex Aiton & Geraniaceae & Alien & Mediterranean Basin & 22 & 53.6 \\
\hline Euphorbia peplus L. & Euphorbiaceae & Alien & Mediterranean Basin & 5 & 12.2 \\
\hline Euphorbia serpens Kunth & Euphorbiaceae & Native & & 7 & 17.0 \\
\hline Festuca arundinacea Schreb. & Poaceae & Alien & Mediterranean Basin & 2 & 4.8 \\
\hline Galinsoga parviflora Cav. & Asteraceae & Native & & 1 & 2.4 \\
\hline Gamochaeta americana (Mill.) Wedd. & Asteraceae & Native & & 9 & 21.9 \\
\hline Geranium robertianum L. & Geraniaceae & Alien & Mediterranean Basin & 1 & 2.4 \\
\hline Hedera helix $\mathrm{L}$. & Araliaceae & Alien & Eurasian & 1 & 2.4 \\
\hline Hirschfeldia incana (L.) Lagr.-Foss. & Brassicaceae & Alien & Mediterranean Basin & 3 & 7.3 \\
\hline Hordeum murinum L. & Poaceae & Alien & Mediterranean Basin & 28 & 68.2 \\
\hline Lactuca sativa $\mathrm{L}$. & Asteraceae & Alien & Asia & 1 & 2.4 \\
\hline Lactuca serriola L. & Asteraceae & Alien & Mediterranean Basin & 2 & 4.8 \\
\hline Lactuca virosa $\mathrm{L}$. & Asteraceae & Alien & Mediterranean Basin & 5 & 12.2 \\
\hline Lamium amplexicaule $\mathrm{L}$. & Lamiaceae & Alien & Mediterranean Basin & 1 & 2.4 \\
\hline Lepidium strictum (S.Watson) Rattan & Brassicaceae & $\begin{array}{l}\text { Native } \\
\text { (endemic) }\end{array}$ & & 26 & 63.4 \\
\hline Lolium perenne L. & Poaceae & Alien & Mediterranean Basin & 7 & 17.0 \\
\hline Lophochloa cristata (L.) Hylander & Poaceae & Alien & Mediterranean Basin & 2 & 4.8 \\
\hline Lotus sp. & Fabaceae & $\begin{array}{l}\text { Not } \\
\text { determined }\end{array}$ & Not determined & 1 & 2.4 \\
\hline Malva neglecta Wallr. & Malvaceae & Alien & Eurasian & 1 & 2.4 \\
\hline Malva nicaeensis All. & Malvaceae & Alien & Mediterranean Basin & 6 & 14.6 \\
\hline Malva parviflora $\mathrm{L}$. & Malvaceae & Alien & Mediterranean Basin & 4 & 9.7 \\
\hline Malva sp. & Malvaceae & $\begin{array}{l}\text { Not } \\
\text { determined }\end{array}$ & Not determined & 17 & 41.4 \\
\hline Malvella leprosa (Ortega) Krapov. & Malvaceae & Native & & 1 & 2.4 \\
\hline Medicago arabica (L.) Huds. & Fabaceae & Alien & Mediterranean Basin & 4 & 9.7 \\
\hline Medicago lupulina L. & Fabaceae & Alien & Mediterranean Basin & 6 & 14.6 \\
\hline
\end{tabular}




\begin{tabular}{|c|c|c|c|c|c|}
\hline SPECIES & & ORIGIN & ORIGINAL DISTRIBUTIONS & $\begin{array}{c}\text { NuMBER } \\
\text { OF PLANTS } \\
\text { COLLECTED }\end{array}$ & $\begin{array}{l}\text { PreVAlENCE } \\
\qquad(\times 100)\end{array}$ \\
\hline Medicago polymorpha $\mathrm{L}$. & Fabaceae & Alien & Mediterranean Basin & 1 & 2.4 \\
\hline Melilotus indicus (L.) All. & Fabaceae & Alien & Mediterranean Basin & 3 & 7.3 \\
\hline Modiola caroliniana (L.) G.Don & Malvaceae & Native & & 3 & 7.3 \\
\hline Nothoscordum gracile (Aiton) Stearn & Alliaceae & Native & & 2 & 4.8 \\
\hline Oxalis arenaria Bertero & Oxalidaceae & Native & & 4 & 9.7 \\
\hline Oxalis corniculata L. & Oxalidaceae & Alien & Mediterranean Basin & 20 & 48.7 \\
\hline Oxalis pes-caprae L. & Oxalidaceae & Alien & Africa & 4 & 9.7 \\
\hline Oxalis rosea Jacq. & Oxalidaceae & Native & & 1 & 2.4 \\
\hline Oxalis sp. & Oxalidaceae & $\begin{array}{l}\text { Not } \\
\text { determined }\end{array}$ & Not determined & 4 & 9.7 \\
\hline Oxalis squamata Zucc. & Oxalidaceae & $\begin{array}{l}\text { Native } \\
\text { (endemic) }\end{array}$ & & 1 & 2.4 \\
\hline Piptatherum miliaceum (L.) Coss. & Poaceae & Alien & Eurasian & 1 & 2.4 \\
\hline Plantago major L. & Plantaginaceae & Alien & Mediterranean Basin & 12 & 29.2 \\
\hline Poa aпnua $\mathrm{L}$. & Poaceae & Alien & Mediterranean Basin & 37 & 90.2 \\
\hline Polygonum aviculare $\mathrm{L}$. & Polygonaceae & Alien & Eurasian & 31 & 75.6 \\
\hline Polygonum sp. & Polygonaceae & $\begin{array}{l}\text { Not } \\
\text { determined }\end{array}$ & Not determined & 1 & 2.4 \\
\hline Portulaca oleracea L. & Portulacaceae & Alien & Eurasian & 2 & 4.8 \\
\hline Ranunculus muricatus L. & Ranunculaceae & Alien & Mediterranean Basin & 1 & 2.4 \\
\hline Ruta chalepensis L. & Rutaceae & Alien & Mediterranean Basin & 1 & 2.4 \\
\hline Sagina apetala Ard. & Caryophyllaceae & Alien & Mediterranean Basin & 8 & 19.5 \\
\hline Senecio vulgaris L. & Asteraceae & Alien & Mediterranean Basin & 3 & 7.3 \\
\hline Sisymbrium irio L. & Brassicaceae & Alien & Mediterranean Basin & 29 & 70.7 \\
\hline Soliva sessilis Ruiz \& Pav. & Asteraceae & Native & & 2 & 4.8 \\
\hline Sonchus asper Garsault & Asteraceae & Alien & Mediterranean Basin & 4 & 9.7 \\
\hline Sonchus oleraceus L. & Asteraceae & Alien & Mediterranean Basin & 10 & 24.3 \\
\hline Sonchus sp. & Asteraceae & Alien & Not determined & 11 & 26.8 \\
\hline Sonchus tenerrimus L. & Asteraceae & Alien & Mediterranean Basin & 7 & 17.0 \\
\hline $\begin{array}{l}\text { Spergularia rubra (L.) J.Presl \& } \\
\text { C.Presl }\end{array}$ & Caryophyllaceae & Alien & Mediterranean Basin & 4 & 9.7 \\
\hline Stellaria media (L.) Vill. & Caryophyllaceae & Alien & Mediterranean Basin & 20 & 48.7 \\
\hline Taraxacum officinale F.H.Wigg. & Asteraceae & Alien & Mediterranean Basin & 26 & 63.4 \\
\hline Trifolium dubium Sibth. & Fabaceae & Alien & Mediterranean Basin & 1 & 2.4 \\
\hline Trifolium repens $\mathrm{L}$. & Fabaceae & Alien & Mediterranean Basin & 12 & 29.2 \\
\hline Triticum aestivum $\mathrm{L}$. & Poaceae & Alien & Eurasian & 1 & 2.4 \\
\hline Urtica urens L. & Urticaceae & Alien & Mediterranean Basin & 12 & 29.2 \\
\hline Veronica arvensis L. & Scrophulariaceae & Alien & Eurasian & 7 & 17.0 \\
\hline Veronica persica Hort. ex Poir. & Scrophulariaceae & Alien & Mediterranean Basin & 2 & 4.8 \\
\hline
\end{tabular}


The taxonomic richness in the samples ranged between 6 and 21 species per transect, corresponding to San Joaquin and Independencia, respectively. No native species was found in the Santiago suburb, while reduced diversity of native species ( $<1$ species per transect) were found in La Cisterna and Renca. The remaining suburbs showed greater numbers of native species per transect (between 1 and 4 species per transect). In regards to alien species, La Cisterna, Santiago Centro, San Joaquín and Renca showed lower species richness per transect $(<7$ species per transect), while the greatest diversity of herbs alien $(>13$ species per transect) were found in Huechuraba, Vitacura, Independence, San Miguel, El Bosque and La Florida. We recorded a positive and significant correlation between native and alien richness $(r=0.44 ; \mathrm{p}<0.05)$.

In terms of bio-geographical origin, $16.5 \%$ (14 species) of the sampled taxa were natives (Fig. 4), whereas $81.1 \%$ were alien plants. Among alien taxa, the Mediterranean Basin represented $54.1 \%$ (46 taxa) of the original distributions recorded (Fig. 4). Taxa from other continents, including America and Australia, represented $1.8 \%$ of all alien species (Fig. 4). But all continents were represented (Table II).

Species distribution between suburbs, presented levels of floristic similarity that oscillated between $J=4.3$ (San Joaquín- La Cisterna, Table II) and $J=66.7$ (San JoaquínCerro Navia, Table II). The similarity cladogram combined with the resample analysis, highlighted two recognisable groups that were not randomly distributed. These groups were distinguished for their similarity value $J=27.1 \%$ combined to a probability $\mathrm{p}<5 \%$ (Fig. 3). The first group included the following suburbs: Santiago Centro, Estación Central, Quinta Normal, Conchalí, San Joaquín, Cerro Navia, Independencia, Recoleta, San Miguel, Pedro Aguirre Cerda, Peñalolén, Macul, Renca, La Granja, Ñuñoa, El Bosque, La Pintana, Lo Prado, and Huechuraba. The second group included La Florida, Providencia, La Reina, Las Condes, and Vitacura (Fig. 3).

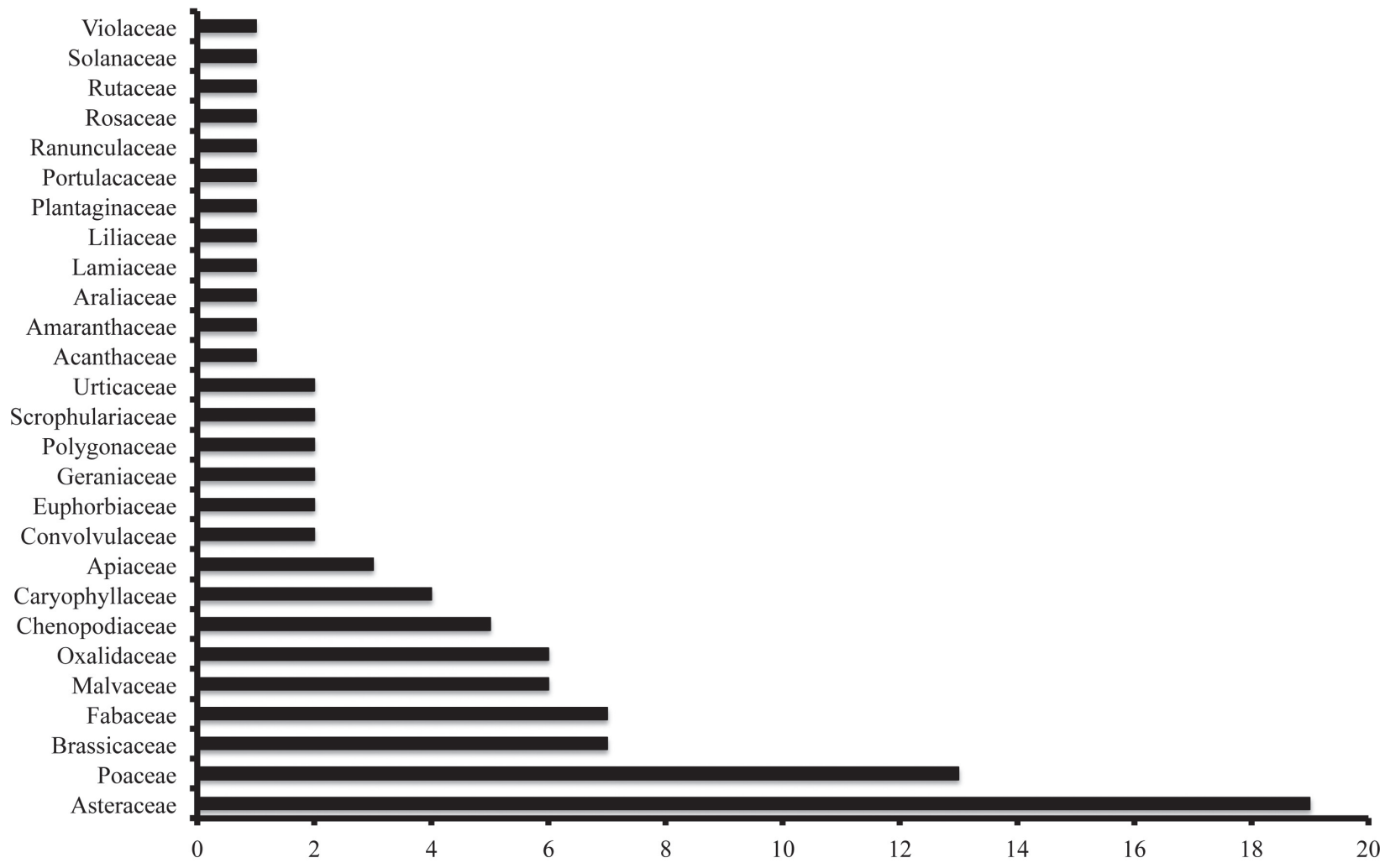

FIGURE 2. Distribution of species richness of the 28 families present in Santiago. Chile.

Figura 2. Distribución de riqueza específica de las 28 familias presentes en Santiago de Chile. 
击

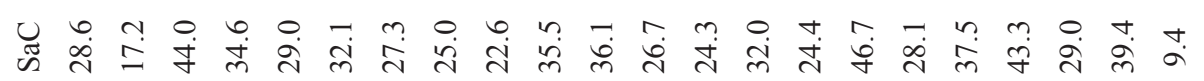

诌

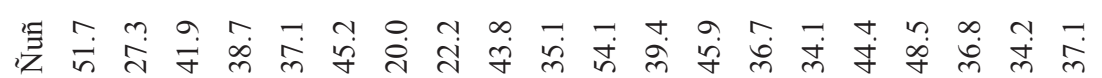

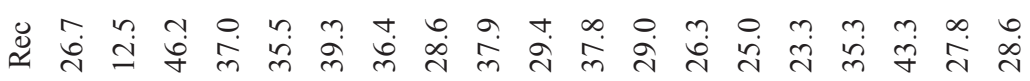

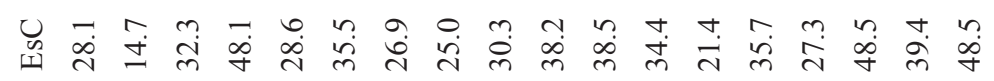

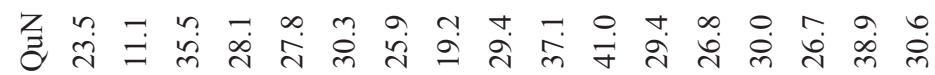

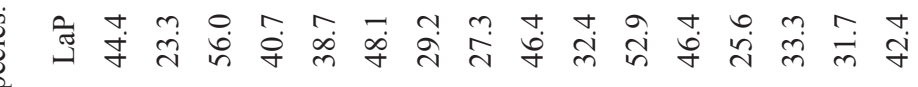

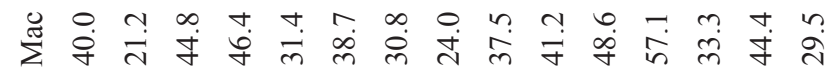

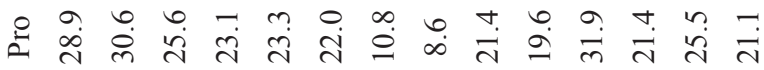

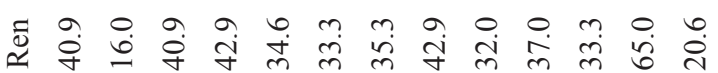

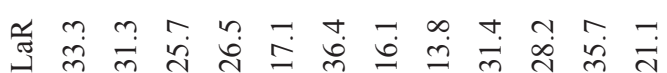

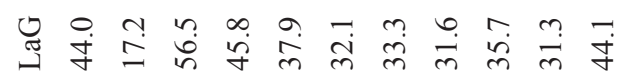

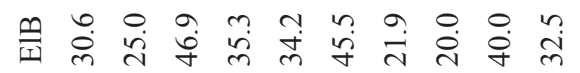

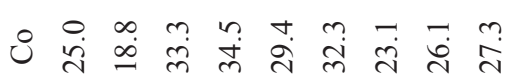

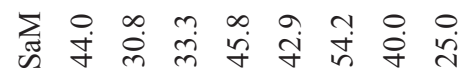

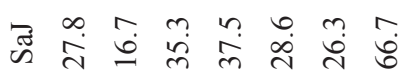

Zु

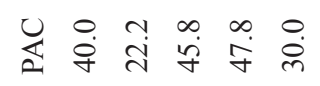

च

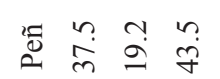

की 0 :

$\vec{i}$

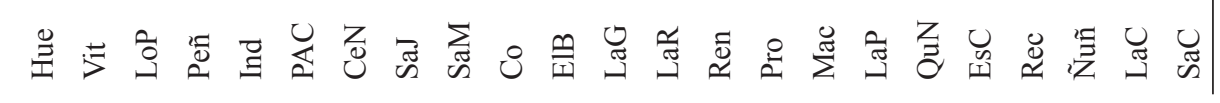




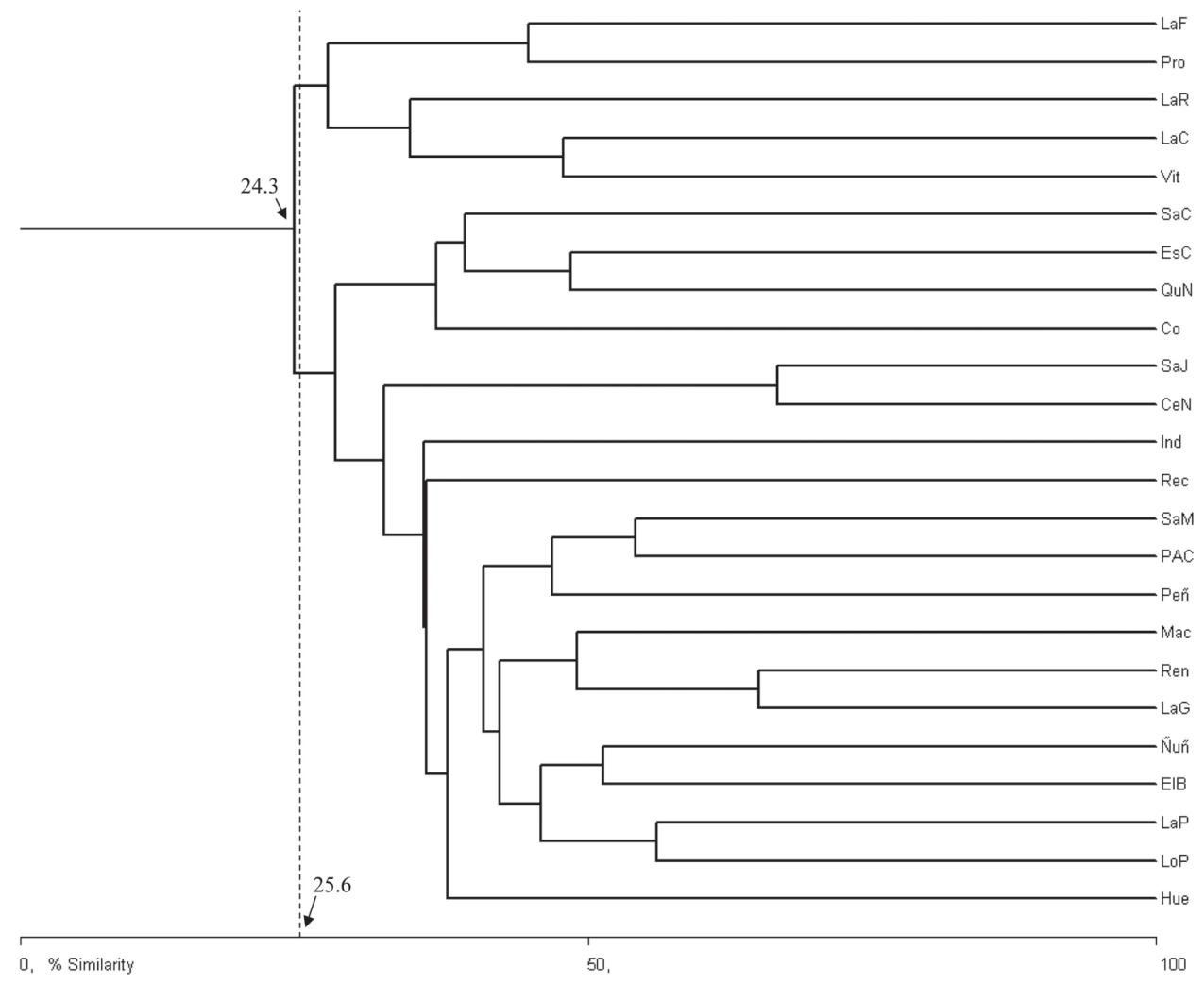

Figure 3. Suburb similarity cladogram in Santiago de Chile (using Jaccard index) according to weed species composition. The segmented line represents the similarity's value (27.1) for which group conglomerates are recognised not to be related to coincidence ( $<<0.05$ ).

Figura 3. Cladograma de similitud para comunas de la ciudad de Santiago de Chile de acuerdo a la composición de especies de malezas. La línea segmentada representa el valor de similitud (27.1) para la cual se reconocen los conglomerados como agrupaciones conformadas más allá del azar $(\mathrm{p}<0.05)$.

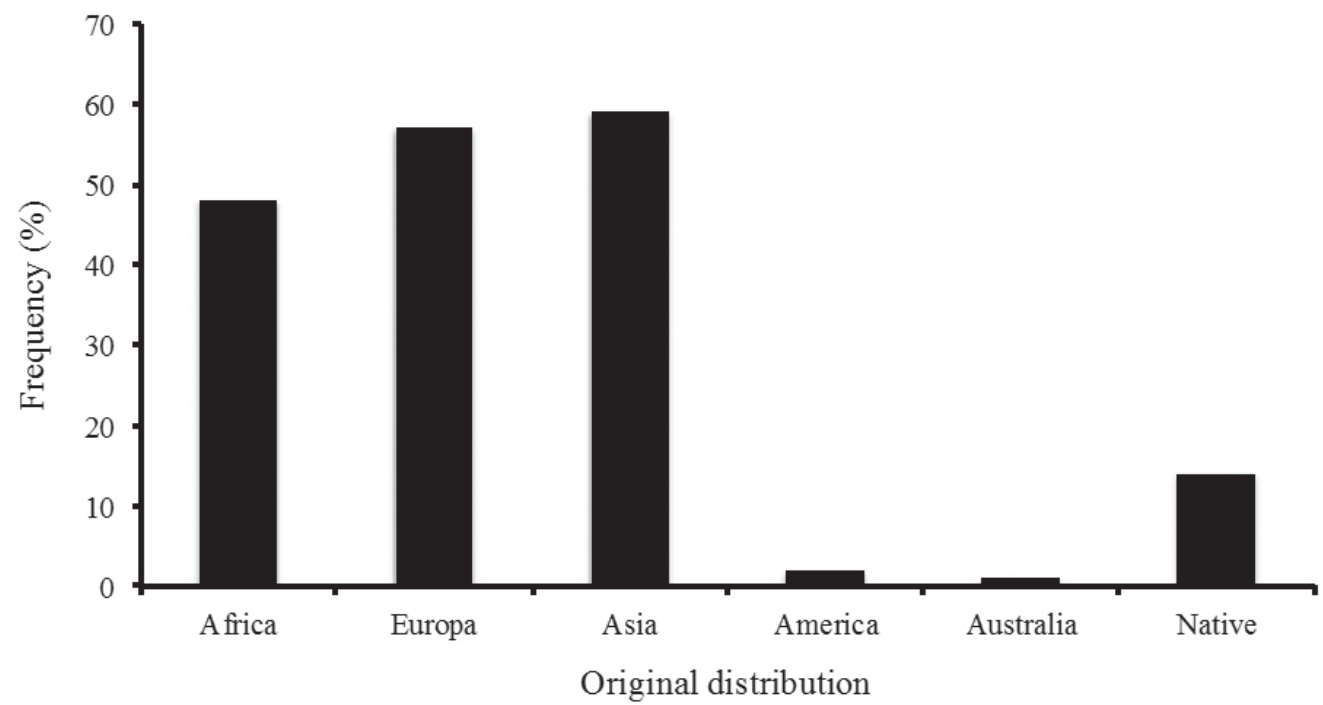

FIGURE 4. Frequency distribution for biogeographic origin of 86 species of herbaceous present in Santiago de Chile.

Figura 4. Distribución de frecuencia para el origen biogeográfico de 86 especies de hierbas presentes en la ciudad de Santiago de Chile. 


\section{DISCUSSION}

The present work contributed to research about native and alien urban plants in the Mediterranean part of Chile. In particular, the results of this study indicated that the urban ruderal flora of Santiago was mainly composed of herbaceous species that have an alien origin, with a low representation of native species. For each native species, we registered approximately 4.9 alien species. Alien species were not only more diverse in terms of number of species but were also more frequent at each sample site (authors, pers. obs.). These observations may be explained by the fact that many alien ruderal species have been introduced in the past for medicinal use, food and/or ornaments (Gay 18451854). The fact that they escaped and apparently aren't used anymore makes it favourable for them to be restricted in ruderal urban environments or at the limit of squares and public and private gardens nowadays (De Vallejo 1980).

The scarcity of studies that describe urban flora in Chile is a notorious fact. Hoffmann (1983) described 105 species of trees in Chilean cities, but did not indicate their distribution, of which $7 \%$ were native and $93 \%$ alien. De la Maza et al. (2002) found that the diversity of species of trees and shrubs in Santiago was correlated with socioeconomic status neighbourhoods. Pauchard et al. (2006) drew attention to the important representation of alien plants in the city of Concepción, soil replacement product to urban use, a situation comparable to Santiago. The high quantity of alien plants in different suburbs of Santiago contrasts with the results of urban studies from other cities (e.g. Brussels, Berlin, Rome), where native species were more diverse than the alien ones (e.g. Albrecht \& Haider 2013, Aronson et al. 2014, Capotorti et al. 2013, Gregor et al. 2012, Knapp et al. 2012, La Sorte et al. 2014, Lososová et al. 2011, Nagendra \& Gopal 2011, Ricotta et al. 2012, Wang et al. 2011). A greater sampling effort is needed to confirm these differences and establish the possible causes. However, we recorded a positive correlation between diversity of native and alien species (by transect), which could be explained considering that the environmental conditions that favour native species also favour alien ones (see Asmus \& Rapson 2014, Sax 2002).

Amongst the alien species, those with Mediterranean origin were dominant, in terms of species richness. This is congruent with the existing studies of Montenegro et al. (1991), Arroyo et al. (2000), Figueroa et al. (2004), Castro et al. (2005), and Fuentes et al. (2013) whom, together, have established that amongst alien species in Chile, those of European-Mediterranean origin are dominant, not only in central Chile but also at the country level. In spite of the absence of others studies, the dominance of species of European origin seems to be a common phenomenon for South America suburbs (i.e. Córdova and Buenos Aires in Argentina; Fortaleza in Brazil), probably as a consequence of Iberian colonisation (MacGregor-Fors \& Ortega-Alvarez 2013).

Intriguingly the analyses of compositional similarity indicated that the spatial distribution of ruderal plants in Santiago was not randomly determined among the suburbs. Basically, two main suburb groups were recognized indicating that herb species are present on some suburbs are not represented on the other one. Reasons for this phenomenon must be further researched but are probably the result of factors such as urban history, socio-economic strata and the use of soil or urban landscaping programs (see Kowarik 2008, Pysek 1998).

Very few studies exist on the ecology of plant species within Chile's urban environments. Floral biodiversity performs important services for the human population; so this is why more studies are essential for understanding how the urbanisation impacts on floral assemblages within cities.

\section{ACKNOWLEDGEMENT}

We deeply thank Alvaro Monzón, Felipe Briceño, Giselle Astorga, Timothy Emery and Alicia Schmidt-Roach for their time reading and correcting the manual and for adding precious ideas. SAC thanks to Proyecto USA 1498.04, Dicyt 021543M and Line 6 of Basal Project CEDENNA FB0807.

\section{REFERENCES}

Albrecht, H.A. \& S. Haider. 2013. Species diversity and life history traits in calcareous grasslands vary along an urbanization gradient. Biodiversity and Conservation 22: 2243-2267.

Aronson, M.F.J., C.H. Nilon, M. Katti, M.A. Goddard, C.A. LepczyK, P.S. Warren, N.S.G. Williams, S. Cilliers, B. Clarkson, C. Dobbs, R. Dolan, M. Hedblom, S. Klotz, J.L. Kooljmans, I. Kühn, I. MacGregor-Fors, M. McDonnell, U. Mörtberg, P. Pyšek, S. Siebert, J. Sushinsky, P. Werner \& M. Winter. 2014. A global analysis of the impacts of urbanization on bird and plant diversity reveals key anthropogenic drivers. Proceedings of the Royal Society, Serie B 281 (1780): 20133330.

Arroyo, M.T.K., C. Marticorena, O. Matthei \& L. Cavieres. 2000. Plant invasions in Chile: present patterns and future predictions. In: H.A. Mooney \& R.J. Hobbs (eds.), Invasive species in a changing world, pp. 385-421. Island Press, Covelo, California.

Asmus, U. \& G.L. RAPSON. 2014. Floristic homogeneity underlines environmental diversification of New Zealand urban areas. New Zealand Journal of Botany 52: 285-303.

Capotorti, G., E. Del Vico, E. Lattanzi, A. Tilia \& L. CelestiGrAPOW. 2013. Exploring biodiversity in a metropolitan area in the Mediterranean region: The urban and suburban flora of Rome (Italy). Plant Biosystems 147: 174-185.

Castro, S.A., J.A. Figueroa, M. Muñoz-Schick \& F.M. Jaksic. 2005. Minimum residence time, biogeographical origin, 
and life cycle as determinants of the geographical extent of naturalized plants in continental Chile. Diversity and Distributions 11: 183-191.

Cursach, J.A., J.R. Rau, C.N. Tobar \& J.A. Ojeda. 2012. Estado actual del desarrollo de la ecología urbana en grandes ciudades del sur de Chile. Revista de Geografía Norte Grande 52: 57-70.

Czech, B. \& P.R. Krausman. 1997. Distribution and causation of species Endangerment in the United States. Science 277: 1116-1117.

De la Maza, C., J. Hernández, H. Bown, M. Rodríguez \& F. Escobedo. 2002. Vegetation diversity in the Santiago de Chile urban ecosystem. Arboricultural Journal 26: 347357.

De Vallejo, A.R. 1980. Malezas de Chile. Instituto de Investigación Agropecuaria, Estación Experimental La Platina. Boletín Técnico 15/16. Santiago, Chile. 47 pp.

Escobedo, F., D. Nowak, J. Wagner, C.L. De la Maza, M. Rodríguez, D. Crane \& J. Hernández. 2006. The socioeconomics and management of Santiago de Chile's public urban forests. Urban Forestry \& Urban Greening 4: 105-114.

Figueroa, J.A., S.A. Castro, P.A. Marquet \& F.M. Jaksic. 2004. Exotic plant invasions to the Mediterranean region of Chile: causes, history and impacts. Revista Chilena de Historia Natural 77: 465-483.

Fuentes, N., A. Pauchard, P. Sánchez, J. Esquivel \& A. Marticorena. 2013. A new comprehensive database of alien plant species in Chile based on herbarium records. Biological Invasions 15: 847-858.

GAY, C. 1845-1854. Historia física y política de Chile. Botánica (Flora chilena). 8 Volumes, 1 Atlas. Santiago and Paris.

Goddard, M.A., A.J. Douglll \& T.G. Benton. 2009. Scaling up from gardens: biodiversity conservation in urban environments. Trends in Ecology and Evolution 25: 90-98.

Gregor, T., D. Bönselb, I. Starke-Ottichb \& G. Zizka. 2012. Drivers of floristic change in large cities: a case study of Frankfurt/Main (Germany). Landscape and Urban Planning 104: 230-237.

HernánDEZ, J. 2007. La situación del arbolado urbano en Santiago. Ambiente Forestal (Chile) 3: 14-16.

Hoffmann, A. 1983. El árbol urbano en Chile. Ediciones Fundación Claudio Gay, Santiago, Chile. 255 pp.

INE. 2002. Instituto Nacional de Estadísticas. URL: http://www. ine.cl. Accessed: September, 2010.

Instituto de Botánica Darwinion. 2011. Flora del Cono Sur. URL: http://www2.darwin.edu.ar/Proyectos/FloraArgentina/ FA.asp Accessed: April, 2010.

JACCARD, P. 1908. Nouvelles recherches sur la distribution florale. Bulletin de la Société Vaudoise des Sciences Naturelles 44: 223-270.

Knapp, S., L. Dinsmore, C. Fissore, S.E. Hobbie, I. Jakobsdottir, J. Kattge, J.Y. King, S. Klotz, J.P. McFadden \& J. Cavender-Bares. 2012. Phylogenetic and functional characteristics of household yard floras and their changes along an urbanization gradient. Ecology 93: S83-S98.

Kowarik, I. \& M. Lippe. 2007. Pathways in Plant Invasions. In: W. Nentwig (ed.), Biological Invasions, pp. 29-47. Springer, Berlin Heidelberg, Germany.

KowARIK, I. 2008. On the role of alien non-native species in urban flora and vegetation. In: J. Marzluff, E. Shulenberger, W. Endlicher, M. Alberti, G. Bradley, C. Ryan, C. Zum-Brunnen \& U. Simon. Urban Ecology (eds.), An international perspective on the interaction between human and nature. pp. 321-338. Springer, New York, USA.

KüHN, I., R. BRANDL \& S. KLOTZ. 2004. The flora of German cities is naturally species rich. Evolutionary Ecology Research 6: 749-764.

La Sorte, F.A. Myla, F. J. Aronson, N.S.G. Williams, L. CelestiGrapow, S. Cilliers, B. D. Clarkson, R. W. Dolan, A. Hipp, S. Klotz, I. KÜHn, P. PyšEK, S. Siebert \& M. Winter. 2014. Beta diversity of urban floras among European and non-European cities. Global Ecology and Biogeography 23: 769-779.

Lososová, Z., Horsák, M., Chytry, M., Cejka, T., Danihelka, J., Fajmon, K., Hájek, O., Juricková, L., K. Kintrová, D. LÁníková, Z. Otypkova, V. Rehorek \& L. Tichy. 2011. Diversity of Central European urban biota: effects of human-made habitat types on plants and land snails. Journal of Biogeography 38: 1152-1163.

Luebert, F. \& P. Pliscoff. 2006. Sinopsis bioclimática y vegetacional de Chile Editorial Universitaria, Santiago de Chile. 316 pp.

MacDonald, R.I., P. Kareva \& R. Forman. 2008. The implications of urban growth for global protected areas and biodiversity conservation. Biological Conservation 141: 1695-1703.

MacGregor-Fors, I. \& R. Ortega-Alvarez. 2013. Ecología urbana: experiencias en America Latina. Available online: www1.inecol.edu.mx/libro_ecologia_urbana. $130 \mathrm{pp}$.

Mack, R.N. \& W.M. Lonsdale. 2001. Humans as global plant dispersers: Getting more than we bargained for. BioScience 51: $95-102$.

Manty, B.F. 2006. Randomization and Monte Carlo methods in biology. Chapman and Hall, New York, USA. 480 pp.

Matthei, O. 1995. Manual de las malezas que crecen en Chile. Alfabeta Impresores, Santiago, Chile. 545 pp.

McGarigal, K., S.A. Cushman \& S.G. Stafford. 2000. Multivariate statistics for wildlife and ecology research. Springer-Verlag, New York, USA. 300 pp.

McKinNeY, M.L. 2006. Urbanization as a major cause of biotic homogenization. Biological Conservation 127: 247-260.

Montenegro, G., S. Teillier, P. Arce \& V. Poblete. 1991. Introductions of plants and animals into the Mediterraneantype climate area of Chile. In: R.H. Groves \& F. di Castri (eds.), Biogeography of Mediterranean invasions, pp. 103114. Cambridge University Press, Cambridge, UK.

Nagendra, H. \& D. Gopal. 2011. Tree diversity, distribution, history and change in urban parks: studies in Bangalore, India. Urban Ecosystems 14: 211-223.

Pauchard, A., M. Aguayo, E. Peña \& R. Urrutia. 2006. Multiple effects of urbanization on the biodiversity of developing countries: The case of a fast-growing metropolitan area (Concepción, Chile). Biological Conservation 127: 272281.

Pickett, S.T.A., M.L. Cadenasso, J.M. Grove, C.H. Nilon, R.V. Pouyat, W.C. Zipperer \& R. Costanza. 2001. Urban ecological systems: linking terrestrial ecological, physical, and socioeconomic components of metropolitan areas. Annual Review of Ecology and Systematics 32: 127-157.

Pysek, P. 1998. Alien and native species in Central European urban 
floras: a quantitative comparison. Journal of Biogeography 25: $155-163$.

RAPOPORT, E. 1993. The process of plant colonization in small settlements and large cities. In: M.J. McDonnell \& S.T.A. Pickett (eds.), Humans as Components of Ecosystems. The Ecology of Subtle Human Effects and Populated Areas. pp. 190-207. Springer, New York, USA.

Reyes-Paecke, S. \& L. Meza. 2011. Jardines residenciales en Santiago de Chile: extensión, distribución y cobertura vegetal. Revista Chilena de Historia Natural 84: 581-952.

Ricotta, C., D. Heathfield, S. Godefroid \& S. Mazzoleni. 2012.
The effects of habitat filtering on the phylogenetic structure of the urban flora of Brussels (Belgium). Community Ecology 13: 97-101.

SAX, D.F. 2002. Native and naturalized plant diversity are positively correlated in scrub communities of California and Chile. Diversity and Distributions 8: 193-210.

Wang, H-F., J. López-Pujol, L.A. Meyerson, J-X. Qiu, X-K. WANG \& Z-Y. OUYANG. 2011. Biological invasions in rapidly urbanizing areas: a case study of Beijing, China. Biodiversity and Conservation 20: 2483-2509.

Recibido: 05.01.15

Aceptado: 02.07.15 